\title{
Commentary: Current status of intratumoral therapy for glioblastoma
}

\author{
Gregory D. Arnone', Matt Wonais², Andreas Linninger ${ }^{1,3}$, Ankit I. Mehta ${ }^{1 *}$ \\ 'Department of Neurosurgery, University of Illinois at Chicago, Chicago, IL 60612, USA \\ ${ }^{2}$ University of Illinois College of Medicine at Chicago, Chicago, IL 60612, USA \\ ${ }^{3}$ Department of Bioengineering, The University of Illinois at Chicago, Chicago, IL, USA
}

Article Info

Article Notes

Received: August 04, 2016

Accepted: September 12, 2016

\section{${ }^{*}$ Correspondence:}

Department of Neurosurgery

University of Illinois at Chicago

912 S. Wood Street (MC-799)

Chicago, IL 60612

Telephone: $312-996-4842$

Fax: 312-996-9018

Email: ankitm@uic.edu

(C) 2016 Mehta Al. This article is distributed under the terms of the Creative Commons Attribution 4.0 International License

\section{Keywords}

convection-enhanced delivery

Nanoparticles

Glioblastoma

Localized drug delivery

Intratumoral therapy
Glial tumors, particularly high grade glioma and glioblastoma multiforme (GBM), continue to pose a significant challenge to neuro-oncologists and neurosurgeons. Despite vigorous research efforts over the past half-century, survival of GBM after maximal surgery, chemotherapy, and radiation therapy remains poor, reaching a median of 14-16 months after diagnosis ${ }^{1}$. New therapies have shown modest increases in median survival, but the majority of patients in these studies still die within two years after diagnosis ${ }^{2,3}$. Drug delivery to the central nervous system (CNS) poses unique challenges. Availability of drugs to cross the blood-brain-barrier (BBB), or more accurately termed blood-CNS barrier, have limited the number, type, and doses of effective therapies against GBM. Various agents, including chemotherapeutics ${ }^{4}$, antiangiogenic drugs $^{5,6}$, immunotoxins ${ }^{7,8}$, viral vectors and gene therapy ${ }^{9}$, among others, have been tried, all with suboptimal results.

Strategies to circumvent the blood-CNS barrier have long been under investigation. Transient osmotic or mechanical disruption of the blood-CNS barrier has been used in order to permit passage of chemotherapeutics into the CNS, though questions regarding lack of specific targeting and safety persist ${ }^{10}$. Localized drug delivery into a postoperative tumor bed also dodges the blood-CNS issue without disruption of normal brain homeostasis, and therefore remains an attractive option for delivery of therapeutics. While ability to cross the blood-CNS barrier has traditionally been critical in designing drugs for use in GBM, the opposite may prove to be most useful for a local delivery strategy. For example, platinum drugs were previously abandoned for treatment of glioma due to their poor passage across the blood-CNS barrier, but are now being re-explored for localized delivery. Adding to the fact that they are not a substrate for principle efflux transporters in the brain, the inability of these agents to cross the blood-CNS barrier may now prove to be an advantage in prolonging the half-life and therapeutic effect of the drug ${ }^{11-13}$.

Gliadel wafers, a carmustine-imbedded polyanhydride copolymer matrix implant, have perhaps been the most successful localized therapy and are FDA approved for newly diagnosed and recurrent glioblastoma, modestly extending overall survival of both groups ${ }^{14}$. Wafer implantation has drawbacks, however, with increased risk of complications such as surgical site breakdown, infection, cerebrospinal fluid leak, brain edema, hydrocephalus, and cyst formation reported in some studies ${ }^{15}$, though not all ${ }^{16,17}$. Nonetheless, tumor recurrence is common even after carmustine 
wafer use, with median survival times of 16.4 and 9.7 months for newly diagnosed and recurrent GBM, respectively. Histological analysis reveals that most tumor recurrence in high-grade glioma is within $2-3 \mathrm{~cm}$ of the tumor margin ${ }^{18,19}$, while drug penetration from surface erosion of the wafer is only within millimeters of the implant ${ }^{20}$. Recent efforts in localized drug delivery have therefore focused on increasing local drug penetration to surrounding brain parenchyma. To that effect, much work has been done regarding understanding the brain extracellular matrix/space (ECS).

In addition to size of molecules, diffusion through the ECS is governed by multiple factors, including ECS local geometry, a net fixed negative charge, binding of antigens to receptors, variable degree of dead space, extracellular matrix molecules (hyaluronan), and intratumoral pressure gradients ${ }^{21}$. In order to promote diffusion of chemotherapeutics, nanocarriers such as liposomes and PEGylated nanoparticles have been developed. These surfaces both protect drugs from being prematurely metabolized, as well as provide charge-shielding from the ECS. In fact, studies on rat and human brain have shown that substances of $114 \mathrm{~nm}$ were able to diffuse through the ECS when protected from the charge influence of the local environment, as opposed to the previous size of 64 $n m$ when unshielded ${ }^{22}$. Another important consideration when studying drug distribution in the brain parenchyma and interstitial space is the rate and mechanism of drug clearance from the brain. Recently, a novel, ubiquitous pathway in the brain has been described that facilitates exchange of cerebrospinal fluid (CSF), interstitial fluid (ISF), and the clearance of solutes from the interstitium. Designated the "glymphatic system", this pathway consists of a para-arterial CSF influx route, a trans-parenchymal pathway that is dependent upon the astrocytic aquaporin-4 (AQP4) water channel for astroglial water transport, and finally a para-venous ISF clearance route ${ }^{23}$. As understanding of this pathway increases, researchers may be able to better predict the clearance of drug from the interstitial space and therefore tailor dosing of local drug depots. Additionally, knowledge of the glymphatic system and its transport channels may ultimately help to develop novel methods to prolong the half-life of chemotherapeutics and nanocarriers.

Another way to promote local drug penetration through the ECS is by convection instead of relying only on bulk diffusion alone. Indeed, convection-enhanced delivery (CED) of a variety of therapeutics has been a popular topic of drug development and delivery for recurrent glioma in the past decade ${ }^{24-26}$. The CED technique utilizes a catheter that is connected to a pump delivering a pressure head behind the infusion of drug. Studies of CED have shown great promise in terms of penetration of therapy through the $\mathrm{ECS}^{27}$. Consequently, CED has become among the most popular vehicles for localized delivery in current time, with 52 studies regarding CED for gliomas published in the past year (pubmed) and 5 ongoing clinical trials of convection enhanced delivery for glioma treatment (clinicaltrials.gov).

Unfortunately, most randomized trials related to CED for glioma have been underwhelming ${ }^{28}$. Perhaps the most notable failure of CED was the PRECISE trial, which did not find any difference in survival when comparing CED of cintredekin besudotox (CB) immunotoxin to gliadel wafers in patients with GBM at first recurrence ${ }^{29}$. A close examination of that study reveals that inconsistent catheter placement, as well as spread of drug into unwanted areas of low resistance, such as subarachnoid space and the ventricles may have reduced the efficacy of CED. With use of currently available image-guidance technology, these issues may be mitigated in future studies. Additionally, novel methods to track CED infusion of drugs may allow real-time feedback of drug distribution. For example, superparamagnetic iron oxide nanoparticles detectable on T2 MRI imaging have recently been incorporated into brain penetrating nanoparticles, providing ability to track therapeutic in the brain for up to one month after administration $^{30}$. Radiolabeled therapeutics are also being used to study the volume of distribution of drug after CED, utilizing interval high-resolution positron emission tomography (PET) scanning to evaluate biologically absorbed doses of radiation in specific anatomical distributions ${ }^{31}$. Still, treatment failure of CED in the PRECISE trial may not be fully explained by suboptimal catheter placement alone. In fact, a retrospective review of catheter placement on patient outcomes in the PRECISE trial showed that overall catheter placement scores did not correlate with local tumor control, progression free survival, or overall survival $^{32}$. The additional issue of infusion backflow also exists in CED, though catheter design has recently been improved with use of multiple ports, backflow-reducing technology, as well as variable injection rates ${ }^{33-36}$. Overall, the pattern of drug dispersal during CED, especially around inhomogeneous peritumoral tissue, has proven to be unpredictable $\mathrm{e}^{37}$, but is steadily improving. Technology development and mathematical modeling may play a future role in determining optimal stereotactic catheter placement, specific to each given tumor and peritumoral environment ${ }^{38-40}$. Aside from the infusion and dispersal issues, CED has an additional disadvantage related to connection of the catheter to an outside system, potentially increasing chance of infection with prolonged or repeated infusions. Additionally, in-hospital monitoring is required during infusion, drastically limiting the effective time that drugs can be administered. Subcutaneous reservoirs of drugs for continuous infusion are being explored, but have yet to be optimized for an intracranial application ${ }^{25,41}$. 
Despite their poor parenchymal penetration, polymer designs for local delivery offer a great advantage over CED in that a local depot of drug can be placed one time, which then releases slowly over a period of days to weeks ${ }^{20,42,43}$. Recently, efforts to pair CED with the use of nanocarriers, including polymers ${ }^{44-46}$, nanoliposomal drug formulations $s^{47-51}$, metallic nanoparticles ${ }^{52}$, liposomally encapsulated radionuclides ${ }^{53}$, and nanodiamonds ${ }^{54}$, among others, have been explored. With this combination of technologies, CED can effectively deliver nanocarriers to the peritumoral area while the nanocarrier vehicle serves as a "convectible depot" of drug that is released to the local environment over time. The characteristics of polymers as biocompatible depots of drug with controlled, predicable release rate makes them most attractive for this purpose, obviating the need for prolonged infusion times or additional infusions. Furthermore, enhancements to nanopolymer design have allowed for development of smaller carriers, approximately $75 \mathrm{~nm}$ in diameter, that are able to penetrate up to sevenfold higher surrounding brain volumes than that of traditional nanopolymer designs of 150-200 $\mathrm{nm}$ diameter ${ }^{55}$. Additionally, novel nanopolymer designs can allow for the controlled release of multiple drugs, each with independent release rates to maximize drug synergy ${ }^{56}$. Polymeric nanoparticles have also been successful for use in aiding non-viral gene delivery for GBM treatment, avoiding the pitfalls of viral vector based therapy, and opening a whole new spectrum of therapies for the modality of local drug delivery ${ }^{57}$. Improved kinetics and duration of drug release, increased efficacy, and decreased neurotoxicity is also possible when nanocarrier platforms are utilized for CED ${ }^{44}$. Another advantage of a nanocarrier platform for CED is the ability to incorporate both therapeutic drugs, as well as the diagnostic imaging probe, creating a "theranostic" particle that can be tracked during infusion ${ }^{58}$. A variety of contrast agents can be incorporated for real-time monitoring of infusate distribution, allowing for adjustment of catheter placement or infusion parameters, if necessary ${ }^{59}$. Real-time CED has shown to be safe, highly predictable, and reproducible in animal experiments, and should greatly enhance the characterization of drug distribution in future patient studies $^{60}$.

Overall, treatment for high-grade glioma remains suboptimal; however, knowing that current technology allows for robust peritumoral application of therapeutics, local drug delivery options remain attractive for delivering optimal drug volume to tumor cells while avoiding systemic side effects and circumventing the issue of the blood-CNS barrier. As nanomedicine continues to evolve and the process of CED is refined, we may see an explosion of hopeful options that could one day turn this devastating disease into a manageable entity.

\section{References}

1. Hart MG, Garside R, Rogers G, Stein K, Grant R. Temozolomide for high grade glioma. Cochrane Database Syst Rev. 2013(4):CD007415.

2. Stupp R, Taillibert S, Kanner AA, et al. Maintenance Therapy With Tumor-Treating Fields Plus Temozolomide vs Temozolomide Alone for Glioblastoma: A Randomized Clinical Trial. JAMA. Dec 15 2015;314(23):2535-2543.

3. Reulen HJ, Poepperl G, Goetz C, et al. Long-term outcome of patients with WHO Grade III and IV gliomas treated by fractionated intracavitary radioimmunotherapy. J Neurosurg. Sep 2015;123(3):760-770.

4. Stupp R, van den Bent MJ, Hegi ME. Optimal role of temozolomide in the treatment of malignant gliomas. Curr Neurol Neurosci Rep. May 2005;5(3):198-206.

5. Khasraw M, Ameratunga MS, Grant R, Wheeler H, Pavlakis N. Antiangiogenic therapy for high-grade glioma. Cochrane Database Syst Rev. 2014(9):CD008218.

6. Popescu AM, Purcaru SO, Alexandru O, Dricu A. New perspectives in glioblastoma antiangiogenic therapy. Contemp Oncol (Pozn). 2016;20(2):109-118.

7. Candolfi M, Kroeger KM, Xiong W, et al. Targeted toxins for glioblastoma multiforme: pre-clinical studies and clinical implementation. Anticancer Agents Med Chem. Oct 2011;11(8):729-738.

8. Calinescu AA, Kamran N, Baker G, Mineharu Y, Lowenstein PR, Castro MG. Overview of current immunotherapeutic strategies for glioma. Immunotherapy. 2015;7(10):1073-1104.

9. Castro MG, Candolfi M, Kroeger K, et al. Gene therapy and targeted toxins for glioma. Curr Gene Ther. Jun 2011;11(3):155-180.

10. Rodriguez A, Tatter SB, Debinski W. Neurosurgical Techniques for Disruption of the Blood-Brain Barrier for Glioblastoma Treatment. Pharmaceutics. 2015;7(3):175-187.

11. Shi M, Fortin D, Sanche L, Paquette B. Convection-enhancement delivery of platinum-based drugs and Lipoplatin(TM) to optimize the concomitant effect with radiotherapy in F98 glioma rat model. Invest New Drugs. Jun 2015;33(3):555-563.

12. White E, Bienemann A, Pugh J, et al. An evaluation of the safety and feasibility of convection-enhanced delivery of carboplatin into the white matter as a potential treatment for high-grade glioma. $J$ Neurooncol. May 2012;108(1):77-88.

13. White E, Bienemann A, Taylor H, Hopkins K, Cameron A, Gill S. A phase I trial of carboplatin administered by convection-enhanced delivery to patients with recurrent/progressive glioblastoma multiforme. Contemp Clin Trials. Mar 2012;33(2):320-331.

14. Chowdhary SA, Ryken T, Newton HB. Survival outcomes and safety of carmustine wafers in the treatment of high-grade gliomas: a metaanalysis. J Neurooncol. Apr 2015;122(2):367-382.

15. Samis Zella MA, Wallocha M, Slotty PJ, et al. Evaluation of postoperative complications associated with repeat resection and BCNU wafer implantation in recurrent glioblastoma. Acta Neurochir (Wien). Feb 2014;156(2):313-323.

16. Xing WK, Shao C, Qi ZY, Yang C, Wang Z. The role of Gliadel wafers in the treatment of newly diagnosed GBM: a meta-analysis. Drug Des Devel Ther. 2015;9:3341-3348.

17. Chaichana KL, Kone L, Bettegowda C, et al. Risk of surgical site infection in 401 consecutive patients with glioblastoma with and without carmustine wafer implantation. Neurol Res. Aug 2015;37(8):717-726.

18. Halperin EC, Bentel G, Heinz ER, Burger PC. Radiation therapy treatment planning in supratentorial glioblastoma multiforme: an analysis based on post mortem topographic anatomy with CT correlations. Int J Radiat Oncol Biol Phys. Dec 1989;17(6):1347-1350. 
19. Gebhardt BJ, Dobelbower MC, Ennis WH, Bag AK, Markert JM, Fiveash JB. Patterns of failure for glioblastoma multiforme following limitedmargin radiation and concurrent temozolomide. Radiat Oncol. 2014;9:130

20. Fleming $A B$, Saltzman WM. Pharmacokinetics of the carmustine implant. Clin Pharmacokinet. 2002;41(6):403-419.

21. Sykova E, Nicholson C. Diffusion in brain extracellular space. Physiol Rev. Oct 2008;88(4):1277-1340.

22. Nance EA, Woodworth GF, Sailor KA, et al. A dense poly(ethylene glycol) coating improves penetration of large polymeric nanoparticles within brain tissue. Sci Transl Med. Aug 29 2012;4(149):149ra119.

23. Iliff JJ, Wang M, Liao $Y$, et al. A paravascular pathway facilitates CSF flow through the brain parenchyma and the clearance of interstitial solutes, including amyloid beta. Sci Transl Med. Aug 15 2012;4(147):147ra111.

24. Healy AT, Vogelbaum MA. Convection-enhanced drug delivery for gliomas. Surg Neurol Int. 2015;6(Suppl 1):S59-67.

25. Ung TH, Malone H, Canoll P, Bruce JN. Convection-enhanced delivery for glioblastoma: targeted delivery of antitumor therapeutics. CNS Oncol. 2015;4(4):225-234.

26. Vogelbaum MA, Aghi MK. Convection-enhanced delivery for the treatment of glioblastoma. Neuro Oncol. Mar 2015;17 Suppl 2:ii3-ii8.

27. Lieberman DM, Laske DW, Morrison PF, Bankiewicz KS, Oldfield EH. Convection-enhanced distribution of large molecules in gray matter during interstitial drug infusion. J Neurosurg. Jun 1995;82(6):10211029.

28. Mehta AI, Linninger A, Lesniak MS, Engelhard HH. Current status of intratumoral therapy for glioblastoma. $J$ Neurooncol. Oct 2015;125(1):1-7.

29. Kunwar S, Chang S, Westphal M, et al. Phase III randomized trial of CED of IL13-PE38QQR vs Gliadel wafers for recurrent glioblastoma. Neuro Oncol. Aug 2010;12(8):871-881.

30. Strohbehn G, Coman D, Han L, et al. Imaging the delivery of brainpenetrating PLGA nanoparticles in the brain using magnetic resonance. J Neurooncol. Feb 2015;121(3):441-449.

31. Luther N, Zhou Z, Zanzonico P, et al. The potential of theragnostic (1) (2)(4)I-8H9 convection-enhanced delivery in diffuse intrinsic pontine glioma. Neuro Oncol. Jun 2014;16(6):800-806.

32. Mueller S, Polley MY, Lee B, et al. Effect of imaging and catheter characteristics on clinical outcome for patients in the PRECISE study. J Neurooncol. Jan 2011;101(2):267-277.

33. Sillay K, Hinchman A, Kumbier L, et al. Strategies for the delivery of multiple collinear infusion clouds in convection-enhanced delivery in the treatment of Parkinson's disease. Stereotact Funct Neurosurg. 2013;91(3):153-161.

34. Schomberg D, Wang A, Marshall H, Miranpuri G, Sillay K. Ramped-rate vs continuous-rate infusions: An in vitro comparison of convection enhanced delivery protocols. Ann Neurosci. Apr 2013;20(2):59-64.

35. Krauze MT, Saito R, Noble C, et al. Reflux-free cannula for convectionenhanced high-speed delivery of therapeutic agents. J Neurosurg. Nov 2005;103(5):923-929.

36. Gill T, Barua NU, Woolley M, et al. In vitro and in vivo testing of a novel recessed-step catheter for reflux-free convection-enhanced drug delivery to the brain. J Neurosci Methods. Sep 30 2013;219(1):1-9.

37. Saucier-Sawyer JK, Seo YE, Gaudin A, et al. Distribution of polymer nanoparticles by convection-enhanced delivery to brain tumors. $J$ Control Release. Jun 28 2016;232:103-112.

38. Smith JH, Humphrey JA. Interstitial transport and transvascular fluid exchange during infusion into brain and tumor tissue. Microvasc Res. Jan 2007;73(1):58-73
39. Haar PJ, Chen ZJ, Fatouros PP, Gillies GT, Corwin FD, Broaddus WC Modelling convection-enhanced delivery in normal and oedematous brain. J Med Eng Technol. Mar 2014;38(2):76-84.

40. Linninger AA, Somayaji MR, Mekarski M, Zhang L. Prediction of convection-enhanced drug delivery to the human brain. J Theor Biol. Jan 7 2008;250(1):125-138.

41. Sonabend AM, Stuart RM, Yun J, et al. Prolonged intracerebral convection-enhanced delivery of topotecan with a subcutaneously implantable infusion pump. Neuro Oncol. Aug 2011;13(8):886-893.

42. Fredenberg S, Wahlgren M, Reslow M, Axelsson A. The mechanisms of drug release in poly(lactic-co-glycolic acid)-based drug delivery systems--a review. Int J Pharm. Aug 30 2011;415(1-2):34-52.

43. Lagarce F, Renaud P, Faisant N, et al. Baclofen-loaded microspheres: preparation and efficacy testing in a new rabbit model. Eur J Pharm Biopharm. Apr 2005;59(3):449-459.

44. Arshad A, Yang B, Bienemann AS, et al. Convection-Enhanced Delivery of Carboplatin PLGA Nanoparticles for the Treatment of Glioblastoma. PLoS One. 2015;10(7): 0132266.

45. Bernal GM, LaRiviere MJ, Mansour N, et al. Convection-enhanced delivery and in vivo imaging of polymeric nanoparticles for the treatment of malignant glioma. Nanomedicine. Jan 2014;10(1):149157.

46. Sawyer AJ, Saucier-Sawyer JK, Booth CJ, et al. Convection-enhanced delivery of camptothecin-loaded polymer nanoparticles for treatment of intracranial tumors. Drug Deliv Transl Res. Feb 1 2011;1(1):34-42.

47. Mendiburu-Elicabe M, Gil-Ranedo J. Combination Therapy of Intraperitoneal Rapamycin and Convection- Enhanced Delivery of Nanoliposomal CPT-11 in Rodent Orthotopic Brain Tumor Xenografts. Curr Cancer Drug Targets. 2015;15(4):352-362.

48. Chen PY, Ozawa T, Drummond DC, et al. Comparing routes of delivery for nanoliposomal irinotecan shows superior anti-tumor activity of local administration in treating intracranial glioblastoma xenografts. Neuro Oncol. Feb 2013;15(2):189-197.

49. Krauze MT, Noble CO, Kawaguchi T, et al. Convection-enhanced delivery of nanoliposomal CPT-11 (irinotecan) and PEGylated liposomal doxorubicin (Doxil) in rodent intracranial brain tumor xenografts. Neuro Oncol. Oct 2007;9(4):393-403.

50. Yamashita Y, Krauze MT, Kawaguchi T, et al. Convection-enhanced delivery of a topoisomerase I inhibitor (nanoliposomal topotecan) and a topoisomerase II inhibitor (pegylated liposomal doxorubicin) in intracranial brain tumor xenografts. Neuro Oncol. Jan 2007;9(1):2028.

51. Noble CO, Krauze MT, Drummond DC, et al. Novel nanoliposomal CPT11 infused by convection-enhanced delivery in intracranial tumors: pharmacology and efficacy. Cancer Res. Mar 1 2006;66(5):2801-2806.

52. Chiarelli PA, Kievit FM, Zhang M, Ellenbogen RG. Bionanotechnology and the future of glioma. Surg Neurol Int. 2015;6(Suppl 1):S45-58.

53. Phillips WT, Goins B, Bao A, et al. Rhenium-186 liposomes as convection-enhanced nanoparticle brachytherapy for treatment of glioblastoma. Neuro Oncol. Apr 2012;14(4):416-425.

54. Xi G, Robinson E, Mania-Farnell B, et al. Convection-enhanced delivery of nanodiamond drug delivery platforms for intracranial tumor treatment. Nanomedicine. Feb 2014;10(2):381-391.

55. Zhou J, Patel TR, Sirianni RW, et al. Highly penetrative, drug-loaded nanocarriers improve treatment of glioblastoma. Proc Natl Acad Sci U $S$ A. Jul 16 2013;110(29):11751-11756.

56. Sharma V, Szymusiak M, Shen H, Nitsche LC, Liu Y. Formation of polymeric toroidal-spiral particles. Langmuir. Jan 10 2012;28(1):729735.

57. Mangraviti A, Tzeng SY, Kozielski KL, et al. Polymeric nanoparticles for 
nonviral gene therapy extend brain tumor survival in vivo. ACS Nano. Feb 24 2015;9(2):1236-1249.

58. Phillips WT, Bao A, Brenner AJ, Goins BA. Image-guided interventional therapy for cancer with radiotherapeutic nanoparticles. Adv Drug Deliv Rev. Sep 30 2014;76:39-59.
59. Mehta AI, Choi BD, Raghavan R, et al. Imaging of convection enhanced delivery of toxins in humans. Toxins (Basel). Mar 2011;3(3):201-206.

60. Krauze MT, Vandenberg SR, Yamashita Y, et al. Safety of real-time convection-enhanced delivery of liposomes to primate brain: a longterm retrospective. Exp Neurol. Apr 2008;210(2):638-644. 